\title{
The Effect of Selection on Genetic Balance when the Population Size is Varying
}

\author{
C. C. Heyde \\ CSIRO Division of Mathematics and Statistics, Canberra, Australia
}

Received August 3, 1976

\begin{abstract}
It is known that a reformulation for variable population size of the classical Sewall Wright model for balance between two genotypes can lead, under some circumstances, to a situation of balanced polymorphism when there is no selection present. In this note it is shown that the presence of selection prohibits the possibility of balance and assures ultimate homozygosity with probability one.
\end{abstract}

\section{INTRODUCTION}

We consider the classical situation of a finite population of haploid individuals in which the generations are nonoverlapping. Suppose that there are $M$ individuals in the population which are either of genotype $a$ or $A$. Suppose also that $a$ has a small selective advantage $\sigma$ over $A$. Then, in the classical Sewall Wright formulation, each generation is produced by binomial sampling with probabilities proportional to the numbers of $a$ and $A$ individuals in the previous generation multiplied by their respective selective values. Indeed, if there are $i$ individuals of type $a$ in a particular generation, the probability that there will be $j$ in the next is

$p_{i j}=\left(\begin{array}{c}M \\ j\end{array}\right)[(1+\sigma) i /(M+\sigma i)]^{j}[(M-i) /(M+\sigma i)]^{M-j}, \quad 0 \leqslant j \leqslant M$.

It is well known that if $X_{n}$ denotes the number of $a$ alleles in the $n$th generation, then $\left\{X_{n}\right\}$ is a finite homogeneous Markov chain with transition probabilities given by (1). Further, the states 0 and $M$ are absorbing while the others are transient, so that the probability of ultimate fixation of one allele or the other is one. This holds for $\sigma \geqslant 0$.

One of the major criticisms levelled at the above model concerns the assumption of a constant population size $M$, which must, in a sense, restrict modes of long-term behaviour. We can, however, following Seneta (1974) and Heyde and Seneta (1975), who have dealt with the case $\sigma=0$, relax this assumption 
in a general way while retaining the essential features of the Wright model. This then allows consideration of, for example, a fluctuating environment.

Let $M_{n}$ be a random variable denoting the population size at time $n$ (with $M_{n}>0$ for all $n$ ) and assume further that (a) the process $\left\{X_{n}, M_{n}, n \geqslant 0\right\}$ is bivariate Markov and (b) the distribution of $X_{n+1}$ given $M_{n+1}, M_{n}, X_{n}$ is binomial with probability given by

$$
\begin{aligned}
&\left(\begin{array}{c}
M_{n+1} \\
j
\end{array}\right)\left[(1+\sigma) X_{n} /\left(M_{n}+\sigma X_{n}\right)\right]^{j}\left[\left(M_{n}-X_{n}\right) /\left(M_{n}+\sigma X_{n}\right)\right]^{M_{n+1}-j} \\
& 0 \leqslant j \leqslant M_{n+1}
\end{aligned}
$$

The question now is to consider the behaviour of $X_{n}$ in this general case. Indeed, if $\sigma=0$ (no selection) it is known that ultimate homozygosity is not in general certain, and a discussion of circumstances under which balanced polymorphism obtains is given in Seneta (1974) and Heyde and Seneta (1975). Roughly speaking, balanced polymorphism can occur if the population size increases sufficiently rapidly. It is the purpose of this note to show that if selection is present $(\sigma>0)$, there must be ultimate homozygosity with probability one. That is, a state of balanced polymorphism is no longer possible, regardless of the behaviour of the population size.

An intuitive explanation for this phenomenon is not difficult to provide. It is elementary and well known that fixation obtains in a deterministic infinite population haploid selection model. Specifically, for genotype $A[a]$ having fitness $1+\sigma[1], A[a]$ will fix if $\sigma>0[\sigma<0]$. On the other hand, for a fixed population size stochastic model (with no mutation) the probability of ultimate fixation is one as in any Markov chain with a bounded number of states. Thus, for large or small populations, the deterministic and stochastic influences both drive towards an outcome of fixation when $\sigma \neq 0$. This situation may be contrasted with the case $\sigma=0$, where the deterministic forces lead to neutral outcomes (all points are invariants).

\section{Mathematical Details}

We confine our attention to the case $\sigma>0$, the case $\sigma=0$ having been discussed previously, as is mentioned above.

Write $F_{n}$ for the $\sigma$ field generated by $\left\{X_{0}, M_{0}, \ldots, M_{n}\right\}, n \geqslant 0$, i.e., the history of the process up to time $n$. Put $Y_{n}=X_{n} / M_{n}$. We can easily see that

$$
E\left(Y_{n+1} \mid F_{n}\right)=\frac{(1+\sigma) Y_{n}}{1+\sigma Y_{n}}=Y_{n}+\frac{\sigma Y_{n}\left(1-Y_{n}\right)}{1+\sigma Y_{n}} \geqslant Y_{n}
$$

so that $\left\{Y_{n}, F_{n}, n \geqslant 1\right\}$ is a submartingale and, since $0 \leqslant Y_{n} \leqslant 1$, the martin- 
gale convergence theorem assures the existence of a random variable $Y$ such that $Y_{n} \rightarrow Y$ a.s. as $n \rightarrow \infty$.

In order to identify $Y$, we take expectations in the above equation to obtain

$$
E Y_{n+1}=E Y_{n}+\sigma E\left[Y_{n}\left(1-Y_{n}\right) /\left(1+\sigma Y_{n}\right)\right] .
$$

Then, letting $n \rightarrow \infty$ and using dominated convergence we obtain

$$
\sigma E[Y(1-Y) /(1+\sigma Y)]=0,
$$

and since $0 \leqslant Y \leqslant 1$ and $\sigma>0$ it follows that $Y(1-Y)=0$ a.s. The introduction of selection thus assures ultimate homozygosity with probability one.

In the classical case, where $M_{n}$ is constant for all $n$, there have been a number of attempts, dating back to Fisher (1930), to obtain accurate bounds on $P(Y=0)=1-P(Y=1)$ (see Moran, 1960 and Arnold, 1968). The problem is much more difficult in the case of stochastically varying $M_{n}$ and we merely note that

$$
E Y_{n} \uparrow E Y=P(Y=1)
$$

as $n \rightarrow \infty$.

\section{REFERENCES}

Arnold, B. C. 1968. A modification of a result due to Moran, J. Appl. Prob. 5, 220-223. Fisher, R. A. 1930. "The Genetical Theory of Natural Selection," Clarendon, Oxford. Heyde, C. C., ANd Seneta, E. 1975. The genetic balance between random sampling and random population size, J. Math. Biol. 1, 317-320.

Moran, P. A. P. 1960. The survival of a mutant gene under selection II, J. Austral. Math. Soc. 1, 485-491.

SENETA, E. 1974. A note on the balance between random sampling and population size, Genetics 77, 607-610. 